\title{
0 Uso de Anti-retrovirais em Gestantes Modifica o Perfil Lipidico?
}

\author{
Do Antiretroviral Agents Modify Lipid Profile in Pregnant Women?
}

Patrícia El Beitune ${ }^{1}$, Geraldo Duarte ${ }^{1}$, José Ernesto dos Santos ${ }^{2}$, Silvana Maria Quintana ${ }^{1}$, Ernesto Antonio Figueiró-Filho ${ }^{1}$, Alessandra Cristina Marcolin ${ }^{1}$

\section{RESUMO}

\begin{abstract}
Objetivo: avaliar o efeito das medicações anti-retrovirais sobre o metabolismo lipidico em
\end{abstract} gestantes portadoras do HIV.

Métodos: estudo prospectivo realizado em 57 gestantes que foram divididas em três grupos: grupo AZT, com 20 pacientes portadoras do HIV em uso de AZT; grupo TT, com 25 pacientes portadoras do HIV em uso de esquemas contendo três anti-retrovirais $(A Z T+3 T C+N F V) e$ grupo controle, com 12 pacientes. Os dados demográficos e antropométricos foram homogêneos entre os grupos estudados. Foram excluidas as pacientes com antecedentes pessoais ou familiares de hiperlipidemia. Amostras sanguineas foram obtidas para avaliação dos lipideos (colesterol total, frações $L D L$ e HDL-colesterol e triglicerideos) em quatro oportunidades durante a gravidez $\left(1^{\circ}=14-20\right.$ semanas; $2^{\circ}=21-26$ semanas; $3^{\circ}=27-32$ semanas e $4^{\circ}$ =33-38 semanas). Os dados foram analisados utilizando-se os testes não paramétricos do $\chi^{2}$, teste de Friedman e teste de Kruskal-Wallis.

Resultados: o uso de anti-retrovirais durante a gestação não induziu diferenças nas taxas do colesterol total e HDL, no entanto, o uso destes agentes esteve associado a elevação de 76,5 $\mathrm{mg} / \mathrm{dL}$ e $84 \mathrm{mg} / \mathrm{dL}$ para $96 \mathrm{mg} / \mathrm{dL}$ e $105 \mathrm{mg} / \mathrm{dL}$ na concentração da fração LDL-colesterol para os grupos AZT e TT, respectivamente $(p<0,01)$. Observou-se associação positiva significante entre os triglicerideos e a carga viral nas gestantes do grupo AZT ( $r=0,53 ; p=0,015)$.

Conclusão: a utilização dos anti-retrovirais durante a gestação eleva significativamente a concentração da lipoproteina $L D L$. Persistem as dúvidas se a gestação potencializa a longo prazo os efeitos dos anti-retrovirais sobre o metabolismo lipidico.

PALAVRAS-CHAVE: Drogas anti-retrovirais. Infecção HIV-1. Infecções na gravidez. Metabolismo lipídico.

\section{Introdução}

A redução objetiva da transmissão vertical do virus da imunodeficiência humana tipo 1 (HIV-1) com o uso de drogas anti-retrovirais caracteriza-

\footnotetext{
${ }^{1}$ Departamento de Ginecologia e Obstetrícia da Faculdade de Medicina de Ribeirão Preto, Universidade de São Paulo ${ }^{2}$ Departamento de Clínica Médica, Divisão de Nutrologia da Faculdade de Medicina de Ribeirão Preto, Universidade de São Paulo Correspondëncia:

Geraldo Duarte

Avenida Bandeirantes, 3900

Hospital das Clínicas da Faculdade de Medicina de Ribeirão Preto, USP

14049-900 - Ribeirão Preto - SP

Fone: (16) 602-2588 / Fax: (16) 633-0946

e-mail: gduarte@fmrp.usp.br
}

Auxílio Financeiro: FAPESP (processo $n^{\circ}$ 01/08450-8) se como um dos mais notáveis avanços para os pesquisadores desse assunto, visto que permite reduzir esta forma de transmissão em mais de $70 \%{ }^{1}$. O uso isolado da zidovudina (AZT) está indicado para gestantes portadoras do HIV-1 visando somente a profilaxia da transmissão vertical deste vírus ${ }^{2}$. Por sua vez, a combinação de três ou mais agentes anti-retrovirais está recomendada durante a gestação quando o tratamento da mãe é necessário em função da carga viral elevada ou do comprometimento orgânico decorrente da infecção, no que pese a associação desses medicamentos com alguns para-efeitos. Dentre esses possiveis para-efeitos destacam-se as alterações sobre o metabolismo lipídico, notadamente com o uso dos inibidores de proteases ${ }^{3-7}$.

A hiperlipidemia é situação conhecida por levar a comprometimento da saúde em humanos ${ }^{8}$. 
O desenvolvimento de aterosclerose é um processo que dura a vida inteira, tendo início na infância. A extensão e gravidade das lesões arteriais aórtica e coronariana em homens e mulheres jovens sem doença cardiovascular clínica são proporcionais às concentrações séricas de colesterol total e colesterol-LDL ${ }^{9}$. Observa-se também que adultos hipercolesterolêmicos têm probabilidade de gerar crianças hipercolesterolêmicas ${ }^{10}$. Adicionalmente, a ocorrência de dislipidemia, em especial as hipertrigliceridemias, durante o período gestacional é fator predisponente para complicações maternas e perinatais, face à elevação da morbidade cardiovascular e pancreatite não raro associadas a essa condição, representadas pela elevação das taxas de mortalidade materna, parto pré-termo e piora dos indicadores de saúde perinatal ${ }^{11,12}$.

Apesar da clara associação entre o uso de inibidores da protease com eventos metabólicos lipídicos adversos, são raros os estudos que enfatizam estas alterações em gestantes utilizando esses fármacos. O presente estudo teve como objetivo portanto, avaliar prospectivamente o efeito dos anti-retrovirais sobre o metabolismo lipídico em gestantes portadoras do vírus HIV-1.

\section{Pacientes e Métodos}

Estudo longitudinal e prospectivo realizado na cidade de Ribeirão Preto, Estado de São Paulo, Brasil, no periodo de setembro de 2001 a março de 2003. O estudo foi realizado com 57 gestantes com idade entre 16 e 43 anos, com gestação única, independente de raça ou paridade. Dessas pacientes, 45 gestantes eram portadoras do HIV-1 e foram selecionadas no momento do seu ingresso na Divisão de Moléstias Infecto-Contagiosas em Ginecologia e Obstetrícia do Hospital das Clínicas da Faculdade de Medicina de Ribeirão Preto da Universidade de São Paulo (HCFMRP-USP). As outras 12 gestantes foram categorizadas como grupo controle (clínica e laboratorialmente normais) e foram selecionadas na Divisão de Pré-Natal de Baixo Risco do HCFMRP-USP. Este estudo foi aprovado pelo Comitê de Ética em Pesquisa do HCFMRP-USP e incluiu somente as pacientes que concordaram em participar do mesmo.

Considerou-se como portadora do HIV-1 a gestante com exames imunoenzimáticos (ELISA) positivos para anticorpos contra o HIV-1 em duas amostras séricas distintas, confirmados pelo teste de Western blot.

O presente estudo selecionou as pacientes portadoras do HIV-1 sem uso prévio de medicações anti-retrovirais. Essas gestantes foram subdivididas em dois grupos, designados grupo AZT e grupo TT. O grupo AZT foi composto de 20 gestantes que preenchiam os requisitos para uso profilático de zidovudina, com contagem de células $\mathrm{TCD}_{4}\left(\mathrm{CD}_{4}\right)$ $>500$ células $/ \mathrm{mL}$ e carga viral $<1.000$ cópias $/ \mathrm{mL}$. O grupo TT foi composto por 25 gestantes com indicação clínica e laboratorial $\left(\mathrm{CD}_{4}<500\right.$ células / $\mathrm{mL}$, independente da carga viral) para receberem terapia anti-retroviral triplice (zidovudina + lamivudina + nelfinavir). Estes critérios são os estabelecidos pelo Perinatal HIV Guidelines Working Group Members quanto ao uso de terapia antiretroviral na gestante ${ }^{13}$.

A dosagem orientada para o grupo AZT foi zidovudina $300 \mathrm{mg} /$ dose em duas tomadas diárias. Para o grupo TT, utilizaram-se zidovudina $300 \mathrm{mg}$, lamivudina $150 \mathrm{mg}$ e nelfinavir $1250 \mathrm{mg}$ por dose em duas tomadas diárias.

Foram excluídas as portadoras de insuficiência renal, hepática, coronariopatia, sindrome nefrótica, aquelas com história pessoal e familiar em primeiro grau de dislipidemia, diabete melito, portadoras de doença de Cushing, hipotiroidismo, doença do armazenamento de glicogênio, lúpus eritematoso sistêmico, porfiria aguda, obstrução biliar extra-hepática e anorexia nervosa. Excluíram-se também pacientes com indice de massa corpórea (IMC) inicial acima de $30 \mathrm{~kg} / \mathrm{m}^{2}$, pacientes sem adesão ao uso regular dos anti-retrovirais e aquelas que utilizaram medicações que induzem anormalidades lipêmicas secundárias, como anti-hipertensivos (diuréticos tiazidicos, furosemida, beta-bloqueadores), corticosteróides, fenotiazinas, cimetidina, ciclosporina e amiodarona.

O valor da mediana da idade materna das pacientes do grupo controle foi 22,5 anos com variação interquartil de 6 anos. No grupo AZT foi de 24 anos ( 7 anos) e no grupo TT foi de 27 anos (6 anos), não sendo detectada diferença estatística entre estas variáveis (teste de Kruskal-Wallis, $\mathrm{p}=0,13$ ). Quanto à raça (branca e não branca), observou-se que 83,50 e $68 \%$ das gestantes respectivamente do grupo controle, AZT e TT apresentaram cor branca (teste do $\chi^{2}, p=0,14$ ). A avaliação dos dados referentes ao tabagismo também não revelou diferenças significativas, observando-se que 91,60 e $80 \%$ das gestantes do grupo controle, AZT e TT respectivamente não eram tabagistas (teste do $\chi^{2}, \mathrm{p}=0,10$ ). Quanto ao aspecto etilismo observou-se que 83,70 e $52 \%$ das gestantes do grupo controle, AZT e TT, respectivamente, não ingeriam bebidas alcoólicas (teste do $\chi^{2}, p=0,14$ ).

As amostras sanguíneas para dosagens séricas dos lípides (colesterol total, frações LDL e HDL-colesterol e triglicerídeos) após jejum de 12-14 
horas foram coletadas por ocasião da primeira consulta e repetidas mais três vezes ao longo da gravidez, em intervalos entre a $14^{a}$ e a $20^{a}$ semana, a $21^{a}$ e a $26^{a}$ semana, a $27^{a}$ e a $32^{a}$ semana e entre a $33^{a}$ e a $38^{\mathrm{a}}$ semana. Adicionalmente, foram avaliados o IMC e o ganho de peso obtidos no momento do ingresso no pré-natal e no momento do parto.

As amostras plasmáticas foram acondicionadas sob refrigeração a $-70^{\circ} \mathrm{C}$ até o seu processamento. O sangue foi coletado em vacutainer sem anticoagulante, processado em duplicatas para dosagem de colesterol e triglicerídeos pelos métodos enzimáticos automatizados, no equipamento Cobas Integra 400 da Roche.

A variabilidade dos niveis séricos dos lipídeos (colesterol total, LDL-colesterol, HDLcolesterol e triglicérídeos) durante a gestação foi analisada considerando-se a mediana e a variação interquartil (respectivamente no $1^{\circ}$ e $3^{\circ}$ quartis). Foram utilizados os testes não paramétricos do $\chi^{2}$, Mann-Whitney, KruskalWallis para comparações múltiplas entre grupos, o teste de Friedman para comparações múltiplas pareadas, com o teste post hoc de Dunn e o teste de correlação de Spearman. Consideraram-se significativas as diferenças com $\mathrm{p}<0,05$. A análise estatística foi realizada utilizando-se o programa computacional SPSS 10.0.

\section{Resultados}

Os dados relativos ao número de linfócitos T- $\mathrm{CD}_{4}{ }^{+}$e da carga viral do HIV-1 encontram-se na Tabela 1. Conforme esperado, a carga viral, inicialmente elevada (14.370 cópias $/ \mathrm{mL}$ ), reduziu significativamente no grupo com tratamento tríplice (TT), chegando a 40 cópias $/ \mathrm{mL}$. Os resultados mostram diferenças significativas entre os grupos AZT e TT (Mann-Whitney, $\mathrm{p}<0,0001)$. Quanto à contagem de linfócitos $\mathrm{T}-\mathrm{CD}_{4}{ }^{+}$, observou-se recuperação significativa nas pacientes do grupo TT, inicialmente com mediana de 399 células $/ \mathrm{mL}$, chegando a 543 células $/ \mathrm{mL}$ no final da gestação. Comparando os grupos TT e AZT, verificou-se diferença significativa destes números entre os dois grupos (teste de Mann-Whitney, $\mathrm{p}<0,0052$ ).

$\mathrm{Na}$ Tabela 2 estão representados os valores das medianas do IMC inicial, do IMC ao término da gestação e do ganho de peso obtido desde o momento do ingresso das gestantes no pré-natal até o momento do parto, nos três grupos estudados. Pode-se observar que o IMC inicial dos grupos controle, AZT e TT foi, respectivamente, $21,9 \mathrm{~kg} / \mathrm{m}^{2}$, $24,3 \mathrm{~kg} / \mathrm{m}^{2}$ e $22,6 \mathrm{~kg} / \mathrm{m}^{2}$ (teste de Kruskal-Wallis, $\mathrm{p}=0,13)$. A avaliação do IMC final também mostrou distribuição uniforme entre os grupos controle, AZT e TT, com medianas de $25,5 \mathrm{~kg} / \mathrm{m}^{2}, 27,7 \mathrm{~kg} / \mathrm{m}^{2}$ e $26,5 \mathrm{~kg} / \mathrm{m}^{2}$, respectivamente (teste de KruskalWallis, $p=0,25)$. Apesar do maior ganho de peso entre as gestantes do grupo controle, não se observaram diferenças entre as pacientes dos três grupos (teste de Kruskal-Wallis, $\mathrm{p}=0,10$ ).

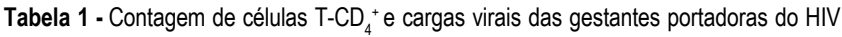
com os valores das medianas e do $1^{\circ} \mathrm{e} 3^{\circ}$ (p25-p75) quartis. Avaliações efetuadas entre a $14^{\mathrm{a}}$ e a $20^{\mathrm{a}}$ semana $\left(1^{\mathrm{a}} \mathrm{CV}\right.$ e $\left.1^{\circ} \mathrm{CD}\right)$ e entre a $33^{\mathrm{a}}$ e a $38^{\mathrm{a}}$ semana $\left(2^{\mathrm{a}} \mathrm{CV}\right.$ e $\left.2^{\circ} \mathrm{CD}{ }_{4}\right)$.

$\mathrm{CV}$ (cópias $/ \mathrm{mL}$ ) $\quad \mathrm{CD}_{4}$ (células $/ \mathrm{mL}$ )

\begin{tabular}{|c|c|c|c|c|}
\hline Grupo & $14-20$ & $33-38$ & $14-20$ & $33-38$ \\
\hline \multirow[t]{2}{*}{ AZT } & 860 & 278 & 692 & 660 \\
\hline & $(600-3.400)$ & $(40-2.923)$ & $(502-895)$ & $(574-862)$ \\
\hline \multirow[t]{2}{*}{ TT } & 14.370 & 40 & 399 & 543 \\
\hline & $(6.726-45.610)$ & $(40-1.499)$ & $(297-494)$ & $(377-689)$ \\
\hline$p$ & $<0,0001^{a}$ & 0,3738 & $<0,0001^{a}$ & $0,0052^{a}$ \\
\hline
\end{tabular}
$\mathrm{CV}=$ Carga viral.

Tabela 2 - Medianas do ganho de peso desde o ingresso no pré-natal até o parto e do índice de massa corpórea (IMC) inicial e ao término da gravidez.

\begin{tabular}{lccc}
\hline Grupo & $\begin{array}{c}\text { Ganho } \\
\text { Peso }\end{array}$ & $\begin{array}{c}\text { IMC inicial } \\
\mathbf{~ k g} / \mathbf{m}^{2}\end{array}$ & $\begin{array}{c}\text { IMC final } \\
\mathbf{~ k g} / \mathbf{m}^{2}\end{array}$ \\
\hline Controle & 10,5 & 21,9 & 25,5 \\
AZT & 8,0 & 24,3 & 27,7 \\
TT & 9,0 & 22,6 & 26,5 \\
p $^{*}$ & 0,1084 & 0,13 & 0,256 \\
\hline
\end{tabular}

${ }^{\star} T$ Teste de Kruskal-Wallis para comparações simultâneas de mais de dois grupos. AZT = Pacientes em uso exclusivo de zidovudina.

$\mathrm{TT}=$ Pacientes em uso de terapia tríplice.

Na Tabela 3 estão os resultados referentes à evolução dos níveis séricos do colesterol total das pacientes dos três grupos avaliados. Observou-se acréscimo do colesterol total ao longo da gestação, independente do regime anti-retroviral utilizado (teste de Friedman, $\mathrm{p}<0,05)$. A comparação entre os niveis séricos de colesterol total entre os grupos nos quatro periodos gestacionais não se traduziu em diferença significativa (teste de Kruskal-Wallis, $\mathrm{p}>0,05$ ).

Os resultados referentes à evolução dos niveis séricos do LDL-colesterol das pacientes dos três grupos avaliados estão na Tabela 4. Observou-se aumento significativo da fração LDL ao longo da gestação apenas nos grupos de portadoras do HIV-1, independente do esquema anti-retroviral utilizado (teste de Friedman, $p<0,01)$. No grupo controle, apesar da elevação dos níveis séricos do LDL-colesterol de $89 \mathrm{mg} / \mathrm{dL}$ para $101 \mathrm{mg} / \mathrm{dL}$, essa variação não foi significativa (teste de Friedman, $\mathrm{p}=0,06$ ). 
Tabela 3 - Valores dos níveis séricos de medianas do colesterol total em $\mathrm{mg} / \mathrm{dL}$ dos três grupos estudados em quatro diferentes períodos gestacionais ( $T 1=14-20$ semanas; $\mathrm{T} 2$ = 21-26 semanas; $\mathrm{T} 3$ = 27-32 semanas; $\mathrm{T} 4$ = 33-38 semanas).

\begin{tabular}{lllllc}
\hline Grupo & \multicolumn{1}{c}{ T1 } & \multicolumn{1}{c}{ T2 } & \multicolumn{1}{c}{ T3 } & \multicolumn{1}{c}{ T4 } & p \\
\hline Controle & 174,5 & 190,5 & 196,5 & $214,5^{\mathrm{a}}$ & $0,0198^{\mathrm{a}}$ \\
AZT & 142,5 & 173 & $181,5^{\mathrm{b}}$ & $190^{\mathrm{b}}$ & $<0,0001^{\mathrm{b}}$ \\
TT & 163 & 183 & 190 & $209^{\mathrm{c}}$ & $<0,0001^{\mathrm{c}}$ \\
${ }^{*} \mathrm{p}$ & 0,068 & 0,306 & 0,830 & 0,340 &
\end{tabular}

arupo Controle - T4 vs T-1: p<0,05 (teste de Friedman, com o teste post hoc de Dunn). ${ }^{\mathrm{b}}$ Grupo AZT - T3 e T4 vs T1: $p<0,0001$ (teste de Friedman e teste post hoc de Dunn). 'Grupo TT - T4 vs T1: $p<0,0001$ (teste de Friedman com o teste post hoc de Dunn).

*Teste de Kruskal-Wallis.

Tabela 4 - Níveis séricos das medianas de LDL-colesterol $(\mathrm{mg} / \mathrm{dL})$ dos três grupos estudados em quatro períodos gestacionais ( $\mathrm{T} 1$ = $14-20$ semanas; $\mathrm{T} 2$ = 21-26 semanas; $\mathrm{T} 3$ = 27-32 semanas; $\mathrm{T} 4$ = 33-38 semanas)

\begin{tabular}{lllllc}
\hline Grupo & T1 & \multicolumn{1}{c}{ T2 } & \multicolumn{1}{c}{ T3 } & \multicolumn{1}{c}{ T4 } & p \\
\hline Controle & 89 & 89 & 101 & 100 & 0,0629 \\
AZT & 76,5 & 85 & 94,5 & $96^{\mathrm{a}}$ & $0,0012^{\mathrm{a}}$ \\
$\mathrm{TT}$ & 84 & 93 & 94 & $105^{\mathrm{b}}$ & $0,0002^{\mathrm{b}}$ \\
${ }^{*} \mathrm{p}$ & 0,1068 & 0,6036 & 0,9146 & 0,7547 &
\end{tabular}

arupo AZT - T4 vs T1: p<0,01(teste de Friedman com o teste post hoc de Dunn). ${ }^{b}$ Grupo TT - T4 vs T1: $p<0,001$ (teste de Friedman e o teste post hoc de Dunn).

*Teste de Kruskal-Wallis.

Observou-se estabilidade dos niveis séricos da fração HDL-colesterol durante a gestação. A mediana da fração HDL entre a $33^{a}$ e a $38^{a}$ semana foi de $59 \mathrm{mg} / \mathrm{dL}$ para o grupo controle, $54,5 \mathrm{mg} / \mathrm{dL}$ para o grupo AZT e $61 \mathrm{mg} / \mathrm{dL}$ para o grupo TT, não se demonstrando influência do período gestacional e dos anti-retrovirais sobre a fração HDL-colesterol (teste de Friedman, $\mathrm{p}>0,65$ ).

Quanto à concentração sérica dos triglicerídeos, observou-se acréscimo mínimo de $50 \%$ dos niveis séricos dessa variável no período entre a 33 e a 38 semana, comparativamente ao período da $14^{\mathrm{a}}$ à $20^{\mathrm{a}}$ semana nos três grupos estudados ao longo da gestação, independente do uso de anti-retroviral (teste de Friedman, $p<0,0001$ ). A comparação entre as gestantes dos três grupos estudados demonstrou valores das medianas dos triglicerídeos de $225 \mathrm{mg} / \mathrm{dL}$ para o grupo controle, $157,5 \mathrm{mg} / \mathrm{dL}$ para o grupo AZT e $250 \mathrm{mg} / \mathrm{dL}$ para o grupo TT, no periodo compreendido entre a $33^{a}$ e a $38^{a}$ semana da gestação (teste de KruskalWallis, $\mathrm{p}=0,09)$.

Na Figura 1 observa-se associação positiva entre os niveis dos triglicerídeos e a carga viral das gestantes portadoras do HIV-1 (coefi-ciente de correlação de Spearman, $\mathrm{p}=0,015$ ) no período de 33 a 38 semanas nas pacientes do grupo AZT. No grupo TT não se verificou a mesma associação (coeficiente de correlação de Spearman, p=0,41).

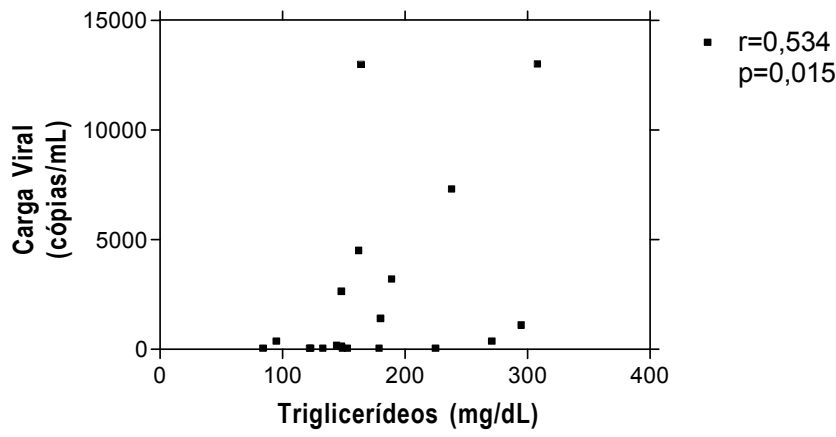

Figura 1 - Associação obtida entre os níveis dos triglicerídeos e a carga viral das gestantes do grupo AZT no período de 33-38 semanas.

\section{Discussão}

Hoje, não existem dúvidas de que a gestação não é razão para postergar a utilização da terapia anti-retroviral se necessária à saúde da mulher. Deve-se considerar, no entanto, dois aspectos: o uso de anti-retroviral como profilaxia para reduzir os riscos de transmissão perinatal do HIV1 e a necessidade de terapia anti-retroviral para tratamento. Para este último objetivo, a utilização de esquemas contendo anti-retrovirais combinados é a opção mais adequada ${ }^{1,2,14,15}$.

Dentre os vários questionamentos relacionados ao uso dos anti-retrovirais combinados durante a gestação, existem dúvidas sobre o papel dos inibidores da protease (IP) na potencialização do risco de dislipidemia associada à gestação ${ }^{2}$. Após pesquisa nos principais bancos de dados médicos e experimentais, não se conseguiu encontrar resultados sobre os efeitos dos anti-retrovirais sobre o metabolismo lipidico, quando utilizados em gestantes. Sob esta ótica, a avaliação da presente casuística é inédita.

A literatura é rica em relatos a respeito dos efeitos da hiperlipidemia na saúde em humanos, aumentando o risco de morbidade cardiovascular ${ }^{5,8,16}$. O objetivo básico da intervenção nas dislipidemias é retardar o desenvolvimento de doença aterosclerótica, reduzindo as contribuições dos fatores circulantes para a progressão da doença. Estimou-se que a redução da concentração sérica de colesterol total de 300 para $200 \mathrm{mg} / \mathrm{dL}$ pode aumentar a expectativa de vida em até três anos e pode retardar a morbidade cardiovascular sintomática em até cinco anos ${ }^{17}$. As condições que favorecem a formação de placa aterosclerótica geralmente refletem o metabolismo anormal das lipoproteínas circulantes ${ }^{18,19}$.

No presente estudo pudemos comparar grupos homogêneos quanto à idade, obesidade, tabagismo, etilismo, história pessoal e familiar de diabete melito e dislipidemia ${ }^{8,9,18,19}$. Adicionalmente, entre os critérios de inclusão optou-se por 
incluir aquele que indicava mulheres com IMC inicial inferior a $30 \mathrm{~kg} / \mathrm{m}^{2}$. Esse estudo selecionou gestantes com IMC inicial de $21,9 \mathrm{~kg} / \mathrm{m}^{2}, 24,3$ $\mathrm{kg} / \mathrm{m}^{2}$ e $22,6 \mathrm{~kg} / \mathrm{m}^{2}$ entre os grupos controle, AZT e TT, respectivamente. O IMC ao término da gestação foi $25,6 \mathrm{~kg} / \mathrm{m}^{2}, 27,7 \mathrm{~kg} / \mathrm{m}^{2}$ e $26,5 \mathrm{~kg} / \mathrm{m}^{2}$ para os mesmo grupos, não havendo diferenças entre os grupos.

Presume-se que o quadro clínico lipidogênico secundário ao uso de anti-retrovirais seja devido à supressão do catabolismo da forma nuclear da proteína ligadora do elemento regulador de esteróis, favorecendo acúmulo dessas proteínas, com conseqüente aumento da biossíntese de colesterol e dos ácidos graxos no figado e resistência insulínica no tecido periférico. Supõe, também que ocorram acréscimos na produção e secreção dos triglicerídeos e lipoproteínas, secundários ao uso dos inibidores da protease devido à inibição da clivagem da apolipoproteina $\mathrm{B}$, bioquimicamente mediada pelas proteasomas ${ }^{20}$. Outro mecanismo infere que possa estar envolvida uma menor expressão dos receptores LDL ${ }^{21}$. No entanto, ainda não se encontram estudos aprofundados sobre a fisiopatologia das alterações induzidas por estes fármacos ${ }^{22-24}$.

A gravidez está associada a alterações fisiológicas importantes do metabolismo dos lipídeos. A primeira é a estimulação da lipólise e da cetogênese, fenômenos progressivos à medida que a gestação evolui. A segunda diz respeito ao aumento gradativo da concentração dos triglicerídeos circulantes com o evolver gestacional, porém, mais constantes no terceiro trimestre da gestação, período em que atingem concentrações de 1,5 a duas vezes acima dos niveis pré-gravídicos ${ }^{25}$.

No presente estudo observou-se elevação dos niveis de colesterol total durante a gestação nos três grupos estudados. Adicionalmente, observou-se elevação significativa dos níveis da fração LDL-colesterol apenas em gestantes portadoras do HIV-1, independente do esquema anti-retroviral utilizado. Os valores individuais dos niveis de triglicerídeos variaram de acordo com o período estudado nos três grupos, à semelhança dos achados observados em outro estudo que apontam aumento em 1,5 a duas vezes nesses niveis ao longo da gestação ${ }^{25}$.

Quando se comparam os valores encontrados nos três grupos, por faixa de idade gestacional, pode-se inferir que não houve diferença significativa entre os parâmetros do lipidograma (colesterol total e frações e triglicerídeos) entre as pacientes do grupo TT e as pacientes do grupo controle. O grupo AZT, apesar de delinear as mesmas tendências de acréscimo dos niveis dos triglicerídeos durante a gestação, exibiu acréscimo de 1,5 vezes em relação aos niveis gestacionais iniciais. A concentração dos triglicerídeos do gru- po TT mostrou-se mais elevada que no Grupo AZT. Este fato pode ser justificado pela associação entre os niveis dos triglicerídeos e a carga viral verificada na presente casuística, achados semelhantes àqueles divulgados por Hadigan et al. ${ }^{23}$. Segundo esses autores, esses resultados sugeriram anormalidades metabólicas que ocorrem à presença do vírus no organismo. Reconhecem, entretanto, que esses achados podem representar o efeito dos fármacos piorando o controle lipídico associado com a melhora clínica e ganho de peso que estas medicações proporcionam. Adicionalmente, no presente estudo não se evidenciou mudança significativa no perfil do HDL durante a gestação, à semelhança dos achados observados em outro estudo, no qual se observou que os inibidores da protease não modificaram o perfil dessa lipoproteína em adultos ${ }^{3}$.

Apesar de os resultados da presente casuística demonstrarem elevação anormal do LDLcolesterol secundária ao uso de anti-retrovirais, deve-se considerar que os benefícios na redução da transmissão vertical do HIV-1 e no controle da saúde materna suplantam os riscos advindos do uso destas medicações. No entanto, persistem as dúvidas se a gestação poderia potencializar os efeitos metabólicos lipídicos dos anti-retrovirais, como o risco de pancreatite e aterosclerose prematura. A prospecção destas mulheres no período puerperal e a longo prazo poderia trazer importantes intervenções neste sentido.

\section{ABSTRACT}

Purpose: to investigate the effect of antiretroviral drugs on the lipid metabolism in HIV-infected pregnant women.

Methods: a prospective study was conducted on 57 pregnant women. The women were divided into three groups: ZDV group, consisting of $20 \mathrm{HIV}$-infected women taking ZDV; TT group, consisting of $25 \mathrm{HIV}$-1-infected women on triple antiretroviral treatment $(\mathrm{ZDV}+3 T C+N F V)$, and control group, consisting of 12 pregnant women considered to be normal from a clinical and laboratory viewpoint. Demographic and anthropometric data were homogeneous. Patients with a personal and family history of hyperlipidemia were excluded. Blood samples were obtained for the determination of fasting lipids (total cholesterol, $L D L$ and $H D L$, and triglycerides) at four periods during pregnancy ( 1 st $=14-20$ weeks; $2 n d=21-26$ weeks; $3 r d=27-32$ weeks and 4 th $=33-38$ weeks). Data were analyzed statistically using the nonparametric $\chi^{2}$, Friedman and Kruskal-Wallis tests.

Results: the use of antiretroviral drugs during pregnancy induced no difference in total or HDL cholesterol but caused an increase from 76.5 and $84 \mathrm{mg} / \mathrm{dL}$ to 96 and $105 \mathrm{mg} / \mathrm{dL}$ in the concentration of the $L D L$ fraction along gestation in $Z D V$ and TT groups, respectively $(p<0.01)$. A positive significant association was observed between triglycerides and viral burden in the ZDV group ( $r: 0.534 ; p=0.015)$. 
Conclusion: Antiretroviral agents during pregnancy increase serum $L D L$-colesterol levels. The risk of pregnancy regarding potentiation of long-term antiretroviral effects on lipid metabolism, remains to be established.

KEYWORDS: Antiretroviral drugs. HIV-1 infection. Infections in pregnancy. Lipid metabolism.

\section{Referências}

1. Connor EM, Sperling RS, Gelber R, et al. Reduction of maternal-infant transmission of human immunodeficiency virus type 1 with zidovudine treatment. Pediatric AIDS Clinical Trial Group Protocol 076 Study Group. N Engl J Med 1994; 331:1173-80.

2. Mofenson LM, Centers for Disease Control and Prevention, U.S. Public Health Service Task Force. Recommendations for the use of antiretroviral drugs in pregnant HIV-1-infected women for maternal health and interventions to reduce perinatal HIV-1 transmission in the United States. MMWR Recomm Rep 2002; 51:1-38.

3. Periard D, Telenti A, Sudre P, et al. Atherogenic dyslipidemia in HIV-infected individuals treated with protease inhibitors. The Swiss HIV Cohort Study. Circulation 1999; 100:700-5.

4. Saves M, Raffi F, Capeau J, et al. Factors related to lipodystrophy and metabolic alterations in patients with human immunodeficiency virus infection receiving highly active antiretroviral therapy. Clin Infect Dis 2002; 34:1396-405.

5. Barthelemy O, Escaut L, Vayre F, et al. Acute coronary syndromes in patients treated with HIV protease inhibitors. Presse Med 2002; 31:343-8.

6. Louie JK, Hsu LC, Osmond DH, Katz MH, Schwarcz SK. Trends in causes of death among person with acquired immunodeficiency syndrome in the era of highly active antiretroviral therapy, San Francisco, 1994-1998. J Infect Dis 2002; 186:1023-7.

7. Stein JH. Dyslipidemia in the era of HIV protease inhibitors. Prog Cardiovasc Dis 2003; 45:293-304.

8. Hopkins PN, Williams RR. A survey of 246 suggested coronary risk factors. Atherosclerosis 1981; 40:1-52.

9. Relationship of atherosclerosis in young men to serum lipoprotein cholesterol concentrations and smoking: a preliminary report from the Pathobiological Determinants of Atherosclerosis in Youth (PDAY) Research Group. JAMA 1990; 264:3018-24.

10.Benuck I, Gidding SS, Donovan M, Traisman ES, Traisman HS. Usefulness of parental serum total cholesterol levels in identifyng children with hypercholesterolemia. Am J Cardiol 1992; 69:713-7.

11. Choy CM, Tam WH, Leung TN. Gestational hyperlipidaemic pancreatitis. BJOG 2002; 109:847-8.
12.Athyros VG, Giouleme OI, Nikolaidis NL, et al. Longterm follow-up of patients with acute hypertriglyceridemia-induced pancreatis. J Clin Gastroenterol 2002; 34:472-5.

13.Public Health Service Task Force Perinatal HIV Guidelines Working Group. Summary of the updated recommendations from the Public Health Service Task Force to reduce perinatal human immunodeficiency virus-1 transmission in the United States. Obstet Gynecol 2002; 99:1117-26.

14.Brocklehurst P, Volmink J. Antiretroviral for reducing the risk of mother-to-child transmission of HIV infection. Cochrane Database Syst Rev 2002; (2):CD003510.

15. Cooper ER, Charurat M, Mofenson L, et al. Combination antiretroviral strategies for the treatment of pregnant HIV-1 infected women and prevention of perinatal HIV-1 transmission. J Acquir Immune Defic Syndr 2002; 29:484-94.

16. Manson JE, Tosteson H, Ridker PM, et al. The primary prevention of myocardial infartion. N Engl J Med 1992; 326:1406-16.

17.Grover SA, Abrahamowicz M, Joseph L, Brewer C, Coupal L, Suissa S. The benefits of treating hyperlipidemia to prevent coronary heart disease. Estimating changes in life expectancy and morbidity. JAMA 1992; 267:816-22.

18.Alva F, Samaniego V, Gonzalez V, Moguel R, Meaney E. Structural and dynamic changes in the elastic arteries due to arterial hypertension and hypercholesterolemia. Clin Cardiol 1993; 16:614-8.

19.Ross R. The pathogenesis of atherosclerosis: a perspective for the 1990's. Nature 1993; 362:801-9.

20.Hui DY. Effects of HIV protease inhibitor therapy on lipid metabolism. Prog Lipid Res 2003; 42:81-92.

21.Petit JM, Duong M, Duvillard L, et al. LDL-receptors expression in HIV-infected patients: relations to antiretroviral therapy, hormonal status, and presence of lipodystrophy. Eur J Clin Invest 2002; 32:354-9.

22. Carr A, Samaras K, Chisholm DJ, Cooper DA. Pathogenesis of HIV-1 protease inhibitor associated peripheral lipodystrophy, hyperlipidaemia, and insulin resistance. Lancet 1998; 351:1881-3.

23.Hadigan C, Miller K, Corcoran C, Anderson E, Basgoz $\mathrm{N}$, Grinspoon S. Fasting hyperinsulinemia and changes in regional body composition in human immunodeficiency virus-infected women. J Clin Endocrinol Metab 1999; 84:1932-7.

24.Tsiodras S, Mantzoros C, Hammer S, Samore M. Effects of protease inhibitors on hyperglicemia, hyperlipidemia, and lipodystrophy: a 5-year cohort study. Arch Intern Med 2000; 160:2050-6.

25.Herrera E, Gomez-Coronado D, Lasuncion MA. Lipid metabolism in pregnancy. Biol Neonate 1987; 51:70-7.

Recebido em: 25/8/2003 Aceito com modificações em: 15/9/2003 